\title{
Radiographic and Histologic Response to Neoadjuvant Radiotherapy in Patients With Soft Tissue Sarcoma
}

\author{
Robert J. Canter, MD¹, Steve R. Martinez, MD, MAS ${ }^{1}$, Robert M. Tamurian, $\mathrm{MD}^{2}$, Maaya Wilton, $\mathrm{MD}^{3}$, \\ Chin-Shang Li, $\mathrm{PhD}^{4}$, Janice Ryu, MD${ }^{5}$, Walter Mak, $\mathrm{MD}^{6}$, Wayne L. Monsky, MD, $\mathrm{PhD}^{6}$, \\ and Dariusz Borys, $\mathrm{MD}^{3}$
}

${ }^{1}$ Division of Surgical Oncology, University of California Davis Medical Center, Sacramento, CA; ${ }^{2}$ Division of Orthopedic Oncology, University of California Davis Medical Center, Sacramento, CA; ${ }^{3}$ Department of Pathology and Laboratory Medicine, University of California Davis Medical Center, Sacramento, CA; ${ }^{4}$ Division of Biostatistics, Department of Public Health Sciences, University of California Davis, Sacramento, CA; ${ }^{5}$ Department of Radiation Oncology, University of California Davis Medical Center, Sacramento, CA; ${ }^{6}$ Department of Radiology, University of California Davis Medical Center, Sacramento, CA

\begin{abstract}
Background. Limited data exist regarding the radiographic and histologic response of soft tissue sarcoma (STS) to neoadjuvant radiotherapy (RT).

Methods. Between February 2000 and January 2009, a total of 25 patients aged $>16$ years with intermediate- or high-grade primary STS of all sites were treated with neoadjuvant RT followed by definitive resection. Patients receiving chemoradiotherapy were excluded. Cross-sectional images obtained before and after RT as well as pathologic specimens were reviewed for maximal change in tumor diameter and percentage tumor necrosis, respectively. Clinicopathologic variables were analyzed for their association with pathologic and radiographic response.

Results. There were 18 extremity (72\%) and 7 retroperitoneal $(28 \%)$ tumors. Median maximal tumor size was $9 \mathrm{~cm}$ (range, $3.3-35 \mathrm{~cm}$ ), and $88 \%$ were of high grade. There were $21 \mathrm{R} 0$ resections $(84 \%)$ and $4 \mathrm{R} 1$ resections $(16 \%)$. Radiographically, the median percentage change in tumor diameter was $0 \%$ (range, -25 to $+86 \%$ ). By Response Evaluation Criteria in Solid Tumors (RECIST), 5
\end{abstract}

Presented in part at the Society of Surgical Oncology 62nd Annual Cancer Symposium, Phoenix, AZ, March 4-8, 2009.

(C) The Author(s) 2010. This article is published with open access at Springerlink.com

First Received: 29 January 2010;

Published Online: 17 June 2010

R. J. Canter, MD

e-mail: Robert.canter@ucdmc.ucdavis.edu patients demonstrated progressive disease, 20 demonstrated stable disease, and 0 demonstrated partial/complete response. The median pathologic percentage tumor necrosis was $30 \%$ (range, 5-100\%). Two tumors (8\%) demonstrated near-complete pathologic response $(\geq 95 \%$ necrosis). Near-complete pathologic response was associated with favorable oncologic outcomes, although these associations were not statistically significant.

Conclusions. Radiologic and near-complete pathologic responses are rare events after preoperative RT for STS. Near-complete pathologic response may be a potentially meaningful surrogate marker for disease outcome and is not predicted by RECIST response. Knowledge of these historical response rates is important for the evaluation of novel neoadjuvant therapies for patients with STS.

External-beam radiotherapy (RT) is an important component of the multimodality treatment of patients with localized soft tissue sarcoma (STS) and has contributed substantially to both effective local disease control and successful limb-sparing surgery. ${ }^{1}$ Since the publication of the landmark National Cancer Institute of Canada randomized trial comparing preoperative to postoperative RT for patients with extremity STS, preoperative RT for STS has been widely accepted. Although oncologic outcomes are essentially equivalent regardless of the timing of RT, many clinicians favor preoperative RT because treatment fields are well-defined and long-term, potentially irreversible, RT-related complications are fewer. ${ }^{2,3}$

Among patients with retroperitoneal sarcoma (RPS), data examining the use of preoperative RT are predominantly 
limited to single-institution phase II trials. ${ }^{4-7}$ Although these trials have reported favorable results, neoadjuvant RT for RPS remains controversial, and a recent randomized phase III trial of preoperative external beam radiation with resection compared to resection alone sponsored by the American College of Surgeons Oncology Group was closed early because of poor accrual.

Nevertheless, interest in preoperative RT for patients with extremity and retroperitoneal STS remains widespread, and ongoing studies are investigating the role of preoperative RT for STS, particularly when administered in combination with novel targeted agents (http://www. clinicaltrials.gov/ct2/results?term=neoadjuvant+sarcoma). Given the interest in investigational treatment approaches combining preoperative RT with novel agents, we sought to determine our historical response rates to neoadjuvant RT monotherapy to provide a benchmark for comparison in future studies. We also sought to determine whether the surrogate end points of pathologic and radiologic response to preoperative RT were associated with definitive oncologic outcome.

\section{PATIENTS AND METHODS}

From February 2000 and January 2009, a total of 45 patients with localized STS were treated with neoadjuvant external beam RT followed by resection with curative intent. These patients were prospectively entered and tracked in a computerized cancer center database. Fourteen patients with low-grade primary tumors and six patients who received combination preoperative chemoradiotherapy were excluded from this analysis. The remaining 25 patients formed the basis of this study.

Preoperative RT was administered in 2-Gy fractions over 25 sessions for a total dose of $50 \mathrm{~Gy}$. Surgical resection was performed approximately 4 to 6 weeks after the completion of RT to allow for acute toxicities to resolve.

After approval for this study by the Institutional Review Board, clinical, pathologic, and treatment data were reviewed and analyzed with respect to percentage pathologic necrosis in the tumor specimen at the time of surgical resection as well as Response Evaluation Criteria in Solid Tumors (RECIST) response on pre- and post-RT crosssectional imaging. Local recurrence-free survival, distant recurrence-free survival, disease-specific survival, and overall survival were also calculated.

Hematoxylin and eosin-stained slides were reviewed in a blinded fashion by a single STS pathologist (D.B.). The percentage of histologically intact tumor and the percentage of necrotic tumor were scored per slide, and the mean percentage of tumor necrosis was calculated for the entire specimen, excluding nonneoplastic tissue. The median number of stained slides examined per patient was 10 (range, 8-18).

Cross-sectional images obtained before and after RT were reviewed by a single musculoskeletal radiologist (W.M.) in a blinded fashion. Among extremity STS, magnetic resonance imaging was used exclusively in all 18 cases. Among retroperitoneal STS, magnetic resonance imaging was used in five of seven cases, computed tomographic scan in one of seven cases, and both modalities in one of seven cases. Tumors were evaluated in three dimensions, and maximal tumor diameter was ascertained. Percentage change in maximal radiographic tumor dimension and RECIST response were calculated (http://ctep. cancer.gov/protocolDevelopment/docs/recist_guideline.pdf).

Data were collected on age, sex, tumor location, histologic type, primary tumor size, maximal tumor diameter, histologic grade, tumor depth, margin status, extent of resection, presence of myxoid histology, and disease recurrence. Major wound complications were classified according to standardized definitions.

Histologic grade was classified by a three-tiered system (grade I through III). Age was determined from the date of diagnosis of the primary tumor. Depth was categorized as either superficial or deep to the investing fascia. By convention, size of the primary tumor was divided into three groups: $\leq 5 \mathrm{~cm},>5 \mathrm{~cm}$ but $\leq 10 \mathrm{~cm}$, and $>10 \mathrm{~cm}$. Sites included extremity (upper at or distal to the shoulder/axilla, and lower at or distal to the buttock/groin) and retroperitoneal.

Histologic diagnosis was assigned by the published criteria of the World Health Organization Classification of Tumors of Soft Tissue and Bone. Margin status was determined either clinically ( $\mathrm{R} 2$ for gross residual tumor left behind) or as part of the histopathologic assessment (R1 for microscopically positive margins, and R0 for microscopically negative margins).

The date of recurrent disease was defined either by biopsy or by the radiographic detection of suspicious lesions when no biopsy was performed. Peritoneal recurrences of intra-abdominal and RPS were considered local recurrences, whereas liver metastases were considered distant recurrences. Follow-up was counted from the date of diagnosis until the date of death or date of last followup. Freedom from local recurrence was counted from the date of resection. Patients who were free from recurrence or death were censored according to the date of their last follow-up.

Summary statistics were reported as mean \pm standard deviation with median (range) where appropriate. Statistical analyses were performed by SAS version 9.1 (SAS Institute, Cary, NC). Nonparametric numerical variables were compared by the Mann-Whitney $U$-test for two groups or the Kruskal-Wallis test for three groups. The Kaplan-Meier approach was used to estimate survival 
curves, and survival differences were analyzed by the $\log$ rank test. ${ }^{8}$ Significance was set at $P<0.05$.

\section{RESULTS}

\section{Patient and Tumor Characteristics}

The clinicopathologic characteristics of the patient cohort are depicted in Table 1 . The median age was 62 years (range, $22-86$ years), and $56 \%$ were women. The site of primary tumor was most commonly the lower extremity (44\%); there was an equal prevalence of upper extremity (28\%) and retroperitoneal (28\%) tumors. The median maximal tumor diameter was $9 \mathrm{~cm}$ (range, 3.3$35 \mathrm{~cm}$ ), $88 \%$ were of high grade, and $96 \%$ were located

TABLE 1 Clinicopathologic characteristics of 25 soft tissue sarcoma patients undergoing preoperative radiotherapy and surgical resection with curative intent

\begin{tabular}{lc}
\hline Characteristic & Value \\
\hline Sex, n (\%) & \\
Male & $11(44)$ \\
Female & $14(56)$ \\
Age at diagnosis (y), median (range) & $62(22-86)$ \\
Site, n (\%) & \\
Lower extremity & $11(44)$ \\
Upper extremity & $7(28)$ \\
Retroperitoneal & $7(28)$ \\
Histology, n (\%) & \\
Malignant fibrous histiocytoma/pleomorphic & $8(32)$ \\
Leiomyosarcoma & $6(24)$ \\
Myxoid/round cell liposarcoma & $5(20)$ \\
Synovial & $3(12)$ \\
Other & $3(12)$ \\
Primary tumor size, n (\%) & \\
$\leq 5$ cm & $2(8)$ \\
$5-10$ cm & $14(56)$ \\
$>10$ cm & $9(36)$ \\
Maximal tumor diameter (cm), median (range) & $9(3.3-35)$ \\
Grade, n (\%) & \\
Intermediate & $22(88)$ \\
High & \\
Depth, n (\%) & \\
Deep & \\
Superficial & \\
Margin status, n (\%) & \\
R0 & \\
R1 & \\
\hline
\end{tabular}

a Includes one mesenchymal chondrosarcoma (4\%), one extraskeletal osteosarcoma (4\%), and one sarcoma, not otherwise specified (4\%) deep to the investing fascia. Seven histologic subtypes were present, including malignant fibrous histiocytoma/ pleomorphic sarcoma (32\%), leiomyosarcoma (24\%), and myxoid/round cell liposarcoma (20\%).

Among 18 patients with extremity tumors, 17 (94\%) were managed successfully with limb-sparing surgery (Table 2). One patient underwent a hip disarticulation for a 24-cm proximal thigh pleomorphic sarcoma involving the posterior and medial compartments from the inferior pubic ramus to the popliteal fossa. An R0 resection was achieved in $84 \%$ of patients, and there were no R2 resections. Major wound complications (as defined by O'Sullivan et al.) occurred in seven patients (28\%). These were more common with lower extremity tumors. ${ }^{2}$

TABLE 2 Treatment and response among 25 soft tissue sarcoma patients undergoing preoperative radiotherapy and surgical resection with curative intent

\begin{tabular}{lc}
\hline Characteristic & Value \\
\hline Extent of resection, n (\%) & $17(68)$ \\
Limb-sparing surgery & $1(4)$ \\
Amputation & $7(28)$ \\
Laparotomy & \\
Major wound complications, ${ }^{\text {a }} \mathrm{n}(\%)$ & $7(28)$ \\
Yes ${ }^{\text {b }}$ & $18(72)$ \\
No & $0(-25$ to +86$)$ \\
Percentage change maximal tumor diameter, & \\
median (range) & \\
RECIST response, $\mathrm{n}(\%)$ & $0(0)$ \\
Complete response/partial response & $20(80)$ \\
Stable disease & $5(20)$ \\
Progressive disease & $30(5-100)$ \\
Percentage pathologic tumor necrosis, median & \\
(range) & \\
Disease recurrence, n (\%) & \\
Local & \\
Distant & $0(0)$ \\
None & $7(28)$ \\
Status at last follow-up, $\mathrm{n}(\%)$ & $18(72)$ \\
No evidence of disease & \\
Alive with disease & $3(12)$ \\
Dead of other causes & \\
Dead of disease & \\
\hline
\end{tabular}

RECIST, Response Evaluation Criteria in Solid Tumors (http:// ctep.cancer.gov/protocolDevelopment/docs/recist_guideline.pdf)

${ }^{\text {a }}$ As defined by O'Sullivan et $\mathrm{al}^{2}$

${ }^{b}$ Includes four patients (16\%) requiring secondary operation for wound repair, two patients $(8 \%)$ requiring readmission for wound care, and one patient (4\%) requiring an invasive procedure for wound management 


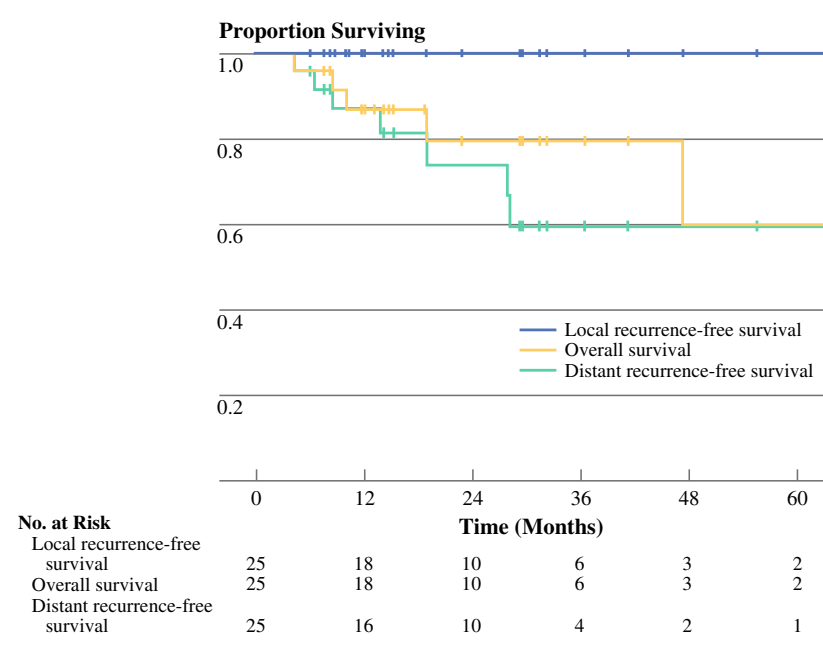

FIG. 1 Kaplan-Meier curve depicting local recurrence-free survival, overall survival, and distant recurrence-free survival for the entire cohort of soft tissue sarcoma patients treated with preoperative radiotherapy followed by resection with curative intent

\section{Oncologic Outcome}

With a median follow-up of 19 months (range, 4-113), 3 -year overall survival, local recurrence-free survival, and distant recurrence-free survival were 80, 100, and 59\%, respectively (Fig. 1). Disease-specific survival was $88 \%$ (data not shown). As depicted in Table 2, seven patients developed distant metastases, 3 of whom died of disease. One patient was rendered free of disease after pulmonary metastasectomy, and three patients received systemic chemotherapy for metastatic disease.

\section{Radiographic and Pathologic Response and Association with Outcome}

As depicted in Table 2, the median percentage change in maximal tumor diameter on serial cross-sectional images

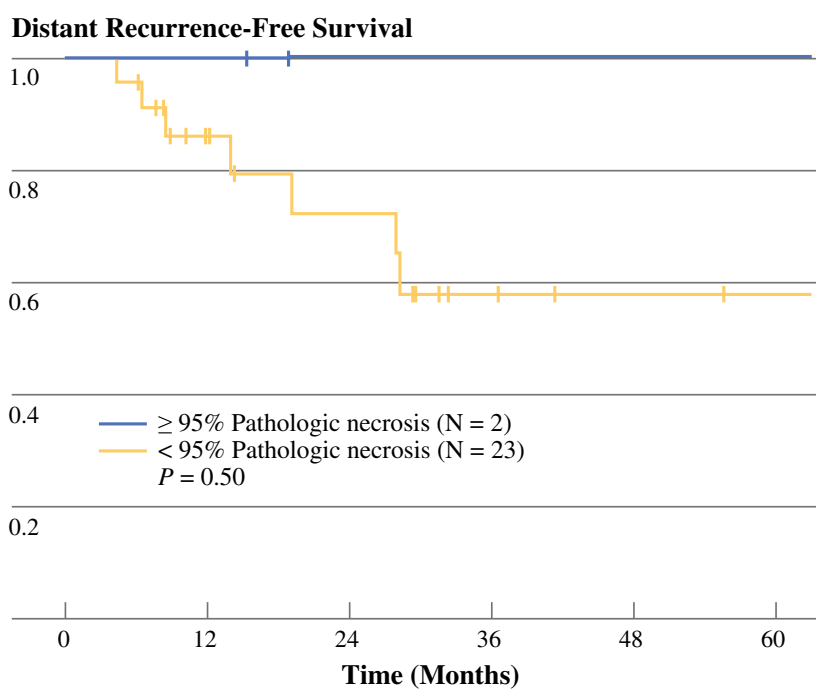

FIG. 3 Kaplan-Meier curve depicting distant recurrence-free survival grouped by presence or absence of near-complete pathologic necrosis in resected surgical specimen ( $\geq 95 \%$ tumor necrosis). Log rank test $P$ value is shown

before and after RT (before resection) was 0\% (range, -25 to $+86 \%)$. This corresponded to 0 complete/partial responses by RECIST, 20 stable disease $(80 \%)$, and 5 progressive disease $(20 \%)$. The distribution of these radiographic responses is shown in Fig. 2a.

The median percentage pathologic necrosis on histologic examination of the resected specimens was $30 \%$ (range, 5-100\%). Two tumors (8\%) demonstrated complete or near-complete pathologic response ( $\geq 95 \%$ necrosis) after preoperative RT. The distribution of tumor necrosis per patient is depicted graphically in Fig. $2 b$.

As depicted in Fig. 3, complete or near-complete pathologic response was associated with favorable distant recurrence-free survival (100\% 3-year event-free survival vs. 59\% 3-year event-free survival). This association, however, was not statistically significant $(P=0.50)$.
FIG. 2 a Waterfall plot of radiographic percentage change in maximal tumor diameter (RECIST response) from initial diagnosis to restaging after preoperative radiotherapy. RECIST, Response Evaluation Criteria in Solid Tumors (http://ctep.cancer.gov/ protocolDevelopment/docs/ recist_guideline.pdf). (b) Waterfall plot of percentage tumor necrosis in resected surgical specimens after preoperative radiotherapy for soft tissue sarcoma a

Radiographic \% Change Maximal Tumor Diameter 100

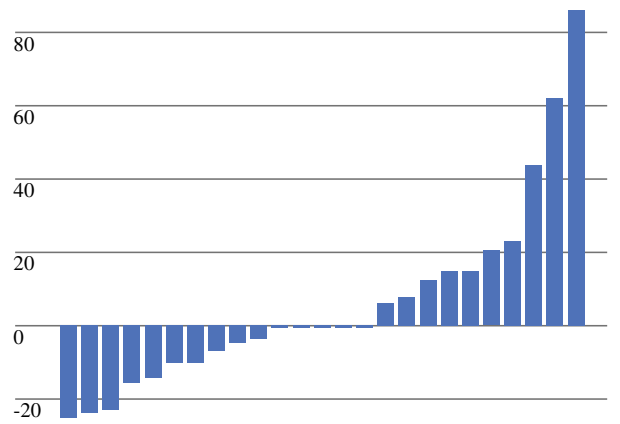

Individual Cases $(\mathbf{N}=\mathbf{2 5})$ b $\%$ Tumor Necrosis

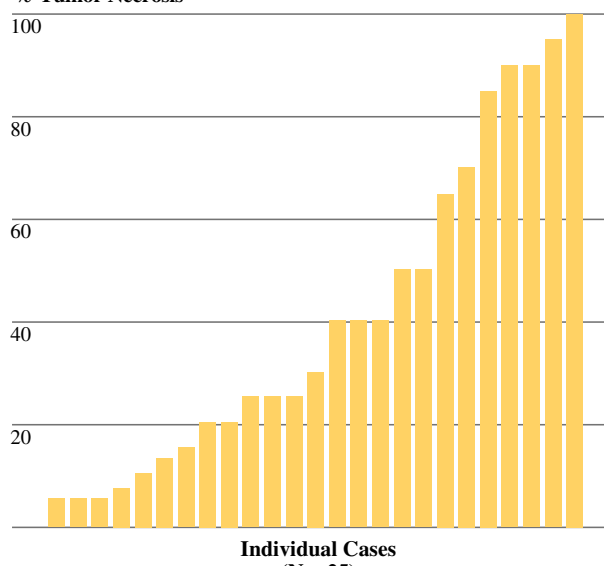
( $N=25)$ 


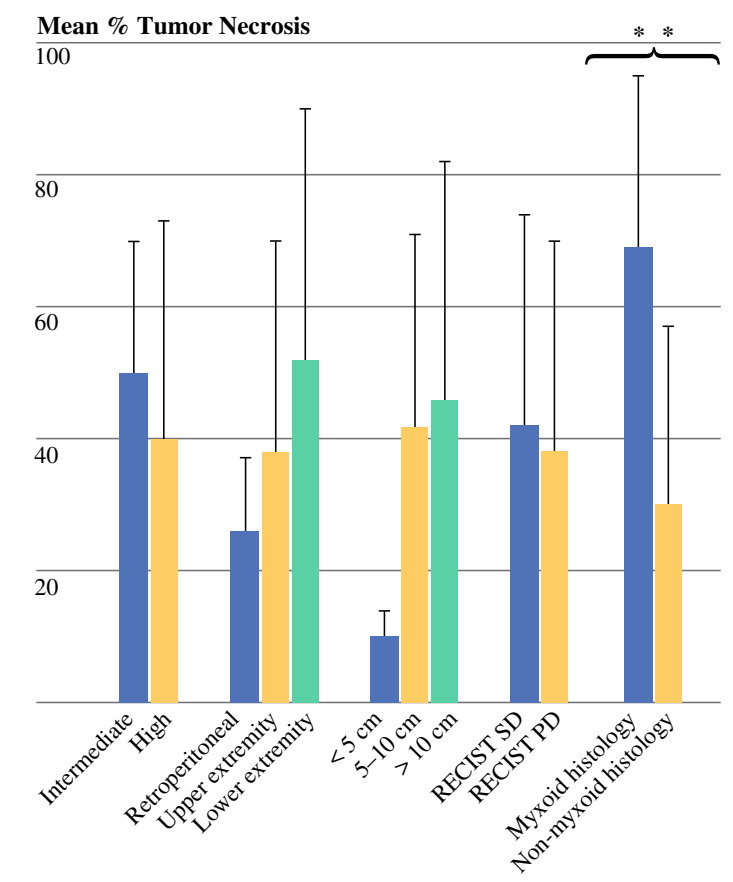

FIG. 4 Bar graph comparing mean percentage tumor necrosis stratified by various clinicopathologic characteristics. $* * P=0.003$ by Mann-Whitney $U$-test. RECIST Response Evaluation Criteria in Solid Tumors; $S D$ stable disease; $P D$ progressive disease. Myxoid histology includes 5 myxoid/round liposarcoma and 2 myxofibrosarcoma

\section{Predictors of Response}

As depicted in Fig. 4, tumors with myxoid histology demonstrated significantly more tumor necrosis after preoperative RT than tumors without myxoid histology (mean percentage necrosis $69 \pm 27 \%$ for myxoid histology vs. $29 \pm 28 \%$ for nonmyxoid histology, $P=0.003$ ). In contrast, none of the following demonstrated statistically significant differences in percentage tumor necrosis after preoperative RT: grade (intermediate grade mean percentage necrosis $50 \pm 20 \%$ vs. high $39 \pm 33 \%, P=$ 0.34 ); location (retroperitoneal mean percentage necrosis $24 \pm 13 \%$ vs. upper extremity $38 \pm 32 \%$ vs. lower extremity $52 \pm 39 \%, P=0.50)$; size $(<5 \mathrm{~cm}$ mean percentage necrosis $10 \pm 4 \%$ vs. $5-10 \mathrm{~cm} 44 \pm 29 \%$ vs. $>10 \mathrm{~cm} 42 \% \pm 40 \%, P=0.33$ ); and RECIST response (stable disease mean percentage necrosis $41 \pm 33 \%$ vs. progressive disease $37 \pm 33 \%, P=0.88$ ).

\section{DISCUSSION}

$\mathrm{RT}$ is an important component of local therapy among patients with STS of the extremity and body wall. ${ }^{1}$ It has been shown to reduce local recurrence rates by $20 \%$ to $25 \%$ relative to radical resection alone. ${ }^{9}$ For patients with RPS, interest in preoperative RT remains widespread because the risk of local treatment failure is substantial and because local failure frequently contributes to patient death. ${ }^{10}$

Despite the frequent administration of preoperative RT for extremity and retroperitoneal STS, there are limited data examining the impact of RT as monotherapy on shortterm end points such as radiographic tumor size or pathologic necrosis in the surgical specimen. For example, no study of preoperative RT to date has reported radiographic and pathologic response rates after treatment among patients with RPS. Among patients with extremity STS, Willett et al. reported $\geq 80 \%$ tumor necrosis in $78 \%$ of preoperatively irradiated patients. ${ }^{11}$ The prevalence of greater degrees of pathologic necrosis in this study may have been the result of twice-daily (total dose 4770 cGy) fractionation in a substantial percentage of patients. Although these patients did experience higher overall amounts of tumor necrosis, there was no correlation between tumor necrosis and distant recurrence-free survival at the $80 \%$ threshold. ${ }^{11}$ Hew et al. observed $\geq 80 \%$ tumor necrosis among $35 \%$ of patients treated with a oncedaily regimen of preoperative $\mathrm{RT}$, a rate which was roughly comparable to that of the present study (25\% of tumors demonstrated $\geq 80 \%$ tumor necrosis). ${ }^{12}$ Similar to Willett et al., Hew et al. did not observe an association between $\geq 80 \%$ pathologic tumor necrosis and distant recurrence-free survival, suggesting that this threshold may not be a biologically significant surrogate end point. ${ }^{12}$

A recent series from de Vreeze et al. demonstrated a $26 \%$ rate of complete pathologic response (100\% necrosis) after preoperative RT. ${ }^{13}$ Although this rate of tumor necrosis is greater than the current study, de Vreeze et al. had a higher proportion of patients with myxoid liposarcoma in their series, and the authors observed complete pathologic response among this entire subgroup of patients. Our findings do confirm the findings of de Vreeze et al. as well as those of other investigators that myxoid histology is consistently associated with favorable tumor necrosis after preoperative RT. ${ }^{14,15}$ Conversely, data regarding the association of tumor size and histologic grade with pathologic response are mixed, and our data indicate a lack of statistically significant association between these other clinicopathologic factors and tumor necrosis. ${ }^{11,12}$

Increasing data suggest that RECIST response is rare after neoadjuvant therapy for STS. ${ }^{16-19}$ Furthermore, changes in radiographic tumor size, as measured by RECIST, also seem to be a poor surrogate of disease control and oncologic outcome. ${ }^{18,20}$ Our findings are consistent with both of these conclusions and reaffirm that novel imaging end points for STS are needed. ${ }^{17,21}$

Although the duration of follow-up was relatively short in this series, preoperative RT followed by wide resection demonstrated excellent local control (100\% local recurrencefree survival), even among patients with radiographically 
progressive disease after preoperative RT. Although nearcomplete pathologic response was associated with favorable oncologic outcome, the rarity of this event ( $8 \%$ of the cohort) rendered our study underpowered to detect differences in survival outcomes, and the survival rates of patients with near-complete pathologic response were not statistically different from those of the entire cohort. Although treatment-induced near-complete pathologic necrosis has been shown to correlate with STS outcome among patients treated with combination preoperative chemoradiation, this association has not been examined in studies of preoperative RT as monotherapy. ${ }^{22,23}$ Because $\mathrm{RT}$ is a local treatment modality, it is conceivable that tumor necrosis after RT monotherapy is not associated with oncologic outcomes (such as overall survival) that are governed by the development of distant disease. However, given the substantial toxicity of combination chemotherapy regimens for STS, there is widespread interest in novel investigational treatments for STS, particularly in combination with preoperative RT (http://www.clinicaltrials.gov/ ct2/results?term=neoadjuvant+sarcoma). This interest in novel neoadjuvant STS therapies highlights the need for additional studies to validate or refute near-complete pathologic response as a potentially important surrogate end point.

In summary, our data demonstrate that radiologic and near-complete pathologic responses are rare events after preoperative RT for STS. Near-complete pathologic response may be a potentially meaningful surrogate marker for disease outcome and is not predicted by RECIST response. Surrogate markers of disease response and prognosis are needed while waiting for longer oncologic follow-up, and knowledge of these historical response rates is important for the evaluation of novel neoadjuvant therapies for patients with STS.

ACKNOWLEDGMENT Statistical support was made possible by grant UL1 RR024146 from the National Center for Research Resources (NCRR), a component of the National Institutes of Health (NIH), and NIH Roadmap for Medical Research. Its contents are solely the responsibility of the authors and do not necessarily represent the official view of NCRR or NIH. Information on Reengineering the Clinical Research Enterprise can be obtained from http://nihroadmap.nih.gov/clinicalresearch/overview-translational.asp.

OPEN ACCESS This article is distributed under the terms of the Creative Commons Attribution Noncommercial License which permits any noncommercial use, distribution, and reproduction in any medium, provided the original author(s) and source are credited.

\section{REFERENCES}

1. Lewis JJ, Brennan MF. Soft tissue sarcomas. Curr Probl Surg. 1996;33:817-72.
2. O'Sullivan B, Davis AM, Turcotte R, et al. Preoperative versus postoperative radiotherapy in soft-tissue sarcoma of the limbs: a randomised trial. Lancet. 2002;359(9325):2235-41.

3. Davis AM, O'Sullivan B, Turcotte R, et al. Late radiation morbidity following randomization to preoperative versus postoperative radiotherapy in extremity soft tissue sarcoma. Radiother Oncol. 2005;75:48-53.

4. Jones JJ, Catton CN, O'Sullivan B, et al. Initial results of a trial of preoperative external-beam radiation therapy and postoperative brachytherapy for retroperitoneal sarcoma. Ann Surg Oncol. 2002;9:346-54.

5. Pawlik TM, Pisters PW, Mikula L, et al. Long-term results of two prospective trials of preoperative external beam radiotherapy for localized intermediate- or high-grade retroperitoneal soft tissue sarcoma. Ann Surg Oncol. 2006;13:508-17.

6. Tzeng CW, Fiveash JB, Heslin MJ. Radiation therapy for retroperitoneal sarcoma. Expert Rev Anticancer Ther. 2006;6:1251-60.

7. Tzeng CW, Fiveash JB, Popple RA, et al. Preoperative radiation therapy with selective dose escalation to the margin at risk for retroperitoneal sarcoma. Cancer. 2006;107:371-9.

8. Mantel N. Evaluation of survival data and two new rank order statistics arising in its consideration. Cancer Chemother Rep. 1966;50:163-70.

9. Yang JC, Chang AE, Baker AR, et al. Randomized prospective study of the benefit of adjuvant radiation therapy in the treatment of soft tissue sarcomas of the extremity. J Clin Oncol. 1998;16:197-203.

10. Canter RJ, Qin LX, Ferrone CR, et al. Why do patients with lowgrade soft tissue sarcoma die? Ann Surg Oncol. 2008;15:3550-60.

11. Willett CG, Schiller AL, Suit HD, et al. The histologic response of soft tissue sarcoma to radiation therapy. Cancer. 1987;60:1500-4.

12. Hew L, Kandel R, Davis A, et al. Histological necrosis in soft tissue sarcoma following preoperative irradiation. J Surg Oncol. 1994;57:111-4.

13. de Vreeze RS, de Jong D, Haas RL, et al. Effectiveness of radiotherapy in myxoid sarcomas is associated with a dense vascular pattern. Int J Radiat Oncol Biol Phys. 2008;72:1480-7.

14. Chung PW, Deheshi BM, Ferguson PC, et al. Radiosensitivity translates into excellent local control in extremity myxoid liposarcoma: a comparison with other soft tissue sarcomas. Cancer. 2009;115:3254-61.

15. Pitson G, Robinson P, Wilke D, et al. Radiation response: an additional unique signature of myxoid liposarcoma. Int $J$ Radiat Oncol Biol Phys. 2004;60:522-6.

16. Schuetze SM, Baker LH, Benjamin RS, et al. Selection of response criteria for clinical trials of sarcoma treatment. Oncologist. 2008;13(Suppl 2):32-40.

17. Stacchiotti S, Collini P, Messina A, et al. High-grade soft-tissue sarcomas: tumor response assessment-pilot study to assess the correlation between radiologic and pathologic response by using RECIST and Choi criteria. Radiology. 2009;251:447-56.

18. Stahl R, Wang T, Lindner LH, et al. Comparison of radiological and pathohistological response to neoadjuvant chemotherapy combined with regional hyperthermia (RHT) and study of response dependence on the applied thermal parameters in patients with soft tissue sarcomas (STS). Int J Hyperthermia. 2009;25:289-98.

19. Ueda T, Naka N, Araki N, et al. Validation of radiographic response evaluation criteria of preoperative chemotherapy for bone and soft tissue sarcomas: Japanese Orthopaedic Association Committee on Musculoskeletal Tumors Cooperative Study. $J$ Orthop Sci. 2008;13:304-12.

20. Benjamin RS. SARC-CTOS imaging symposium: introduction to the problem from a clinical perspective. Oncologist. 2008; 13(Suppl 2):1-3. 
21. Evilevitch V, Weber WA, Tap WD, et al. Reduction of glucose metabolic activity is more accurate than change in size at predicting histopathologic response to neoadjuvant therapy in highgrade soft-tissue sarcomas. Clin Cancer Res. 2008;14:715-20.

22. Eilber FC, Rosen G, Eckardt J, et al. Treatment-induced pathologic necrosis: a predictor of local recurrence and survival in patients receiving neoadjuvant therapy for high-grade extremity soft tissue sarcomas. J Clin Oncol. 2001;19:3203-9.

23. Macdermed DM, Miller LL, Peabody TD, et al. Primary tumor necrosis predicts distant control in locally advanced soft-tissue sarcomas after preoperative concurrent chemoradiotherapy. Int $J$ Radiat Oncol Biol Phys. 2010;76:1147-53. 